\begin{tabular}{|c|c|}
\hline $\begin{array}{l}\text { Journal of } \\
\text { Radiotherapy } \\
\text { in Practice }\end{array}$ & $\begin{array}{l}\text { Yournal of Radiotherapy in Practice (2010) } \\
\mathbf{9}, 185-197 \\
\text { ○ Cambridge University Press } 2010 \\
\text { doi:10.1017/S1460396910000385 }\end{array}$ \\
\hline
\end{tabular}

\title{
Abstracts
}

\section{Abstracts from papers presented at the 5th Radiotherapy in Practice Conference, Sheffield Hallam University, Sheffield, UK}

\section{8-10th 0ctober 2010}

PRONE BREAST IRRADIATION:

TURNING THINGS UPSIDE DOWN IN WINDSOR

\section{Carol Agappito}

Windsor Regional Cancer Center, Ontario, Canada

The irradiation of large pendulous breasts is always a challenge in radiation therapy departments. Many immobilization techniques have been tried, and with IMRT/control points, there are more options available for attempting to create dose distributions that are more homogenous and are more reproducible on a daily basis.

At the Windsor Regional Cancer Centre (WRCC), we have attempted to immobilize large pendulous breasts in the supine position using a variety of commercial and in-house methods, with varying degrees of success, but the underlying problem is always the time for setup and reproducibility of the breast position, and thereby the dose distribution.

Breast conservation therapy is a safe, effective alternative to mastectomy for many women with newly diagnosed breast cancer. This approach involves local excision of the malignancy with tumour-free margins, followed by 5-7 weeks of external beam whole breast radiotherapy to minimise the risk of an in-breast tumour recurrence.
Unfortunately breast conservation therapy is often considered to be contraindicated for large breasted women based on studies that have shown increase chronic fibrosis, increased radiodermatitis, poorer cosmetic outcome, marked dose inhomogeneity $(>10 \%)$ and bolus effect of the inframammary fold.

By treating the patient in the prone position, many of the complications with immobilization and reproducibility are minimized. In my presentation, I will outline our prone breast technique, dose distributions and dosimetric data acquired. Other findings including patient tolerance and acute cosmetic outcomes will be discussed.

\section{PROGRESS WITH IMPLEMENTATION OF THE NATIONAL RADIOTHERAPY ADVISORY GROUP RECOMMENDATIONS: A SOCIETY OF RADIOGRAPHERS PERSPECTIVE Charlotte Beardmore, Sarah James}

Professional Officers for Radiotherapy, The Society and College of Radiographers, UK

This presentation will outline progress with implementation of the National Radiotherapy Advisory Group recommendations 'Developing World Class Radiotherapy Services' ${ }^{1}$ which 
were endorsed within the Cancer Reform Strategy. ${ }^{2}$

The National Radiotherapy Advisory Group (NRAG) report was submitted to Ministers over 3 years ago.

Key points from this report:

- The need for radiotherapy was underestimated in the past

- Demand is set to grow over the next 10 years

- There is currently a 2.5 -fold variation in radiotherapy activity between cancer networks which cannot be attributed to differences in levels of need

- On average 30,000 fractions are currently being delivered per million population, with a range of 17,000 to 48,000 fractions per million between networks

- By 2016 it is estimated that there will be a need for around 54, 000 fractions per million population requiring more staff and linear accelerators

- The NHS needs to make best use of existing staff and equipment. Across a radiotherapy department an average output of 8,000 fractions per linac per annum should be achievable immediately working towards at least 8,700 fractions by 2016

- There is a clear need to collect better data on radiotherapy activity.

\section{Cancer Reform Strategy $2007^{2}$}

The report included a number of recommendations identifying both investment in capacity and workforce as essential requirements in order to deliver world class radiotherapy services.

The Society of Radiographers as key stakeholders within NRAG and NRIG (National Radiotherapy Implementation Group) continue to support the development of radiotherapy services in line with the original recommendations.
This paper will discuss, from our perspective as stakeholders the progress made to date In England, over the past 3 years. Importantly the learning is being shared across the countries of the UK, and the mechanism for this will be referred to during the presentation.

\section{References}

1. NRAG. Radiotherapy: developing a world class service for England Report to Ministers from National Radiotherapy Advisory Group, 2007.

2. Department of Health. The Cancer Reform Strategy. London: Department of Health, 2007.

\section{THE ROLE OF THE ADVANCED \\ PRACTITIONER IN THE \\ SUCCESSFUL IMPLEMENTATION \\ AND DELIVERY OF IMAGE- \\ GUIDED BRACHYTHERAPY}

\section{Katie Bradshaw}

Addenbrooke's Hospital, Cambridge, UK

This paper explores the significance of the Advanced Practitioner role, during service redesign and within delivery of the IGBT pathway; identifying how the role facilitates effective communication and functions as an intermediary between the multidisciplinary team.

\section{Background}

For bulky cervical tumours, MRI-based intracavitary techniques with the addition of interstitial needles, has been shown to give superior local control rates and reduce toxicity. In light of this evidence, Cambridge University Hospital became the first centre in the UK to implement this relatively new concept in 2009 .

\section{Discussion}

The goal for the gynaecological oncology team was straightforward; Implement IGBT, gain superior local control rates, reduce long-term 
morbidity and provide seamless, patient focussed care.

Implementing successful service redesign represents a huge challenge for all staff involved. The author's role has been identified as pivotal, particularly within development of the following key areas:

- Clear role definition

- Staff involvement

- Role development and extension

- Education and training provision

- Overcoming resistance to change

Delivery of the brachytherapy pathway is an increasingly complex process and a daunting experience for many patients. Involvement of the Advance Practitioner facilitates provision of effective information and support throughout the patient pathway, ensuring patients receive a high standard of care and the pathway remains, at all times focussed around the patient needs.

\section{Summary}

Implementation and smooth delivery of IGBT has been overwhelmingly successful at the author's cancer centre and this paper demonstrates how the role of the advanced practitioner has been instrumental.

\section{DEVELOPMENT OF ADVANCED \\ PRACTICE FOR RADIATION THERAPISTS IN NEW ZEALAND}

\section{Karen Coleman}

University of Otago, New Zealand

\section{Background}

A recent New Zealand survey showed overwhelming support for radiation therapist (RT) role extension, especially in the areas of RTled treatment review clinics, breast mark-up and patient education.
Aim

To evaluate the 'in house' treatment review programme for RTs at Dunedin Hospital. This programme was designed to expand the clinical experience of RTs. The evaluation considered the benefits and challenges of the training and investigated whether a formal postgraduate study may support this clinical programme.

\section{Method}

Three semi-structured interviews of participants were conducted, transcribed and subjected to a thematic content analysis by an independent researcher.

\section{Results}

The beneficial aspects of participating in the in house' treatment review programme included; increased knowledge, satisfaction with patient care, increased department efficiency and strengthened relationships within the department. Challenging aspects of participation included the intensity of the role and adequate supervision/mentoring. Information from the survey, 'in house' evaluation and communication with overseas universities has led the University of Otago to develop two postgraduate papers in RT Advanced Practice. These papers will fit with Health Sciences framework and provide the basis of an academic underpinning of the RT Advanced practitioner role.

DEVELOPMENT OF A DECISION AID

AND FIRST IMPRESSIONS: A PILOT

STUDY FOR OLDER WOMEN WITH

STAGE 1 HORMONE SENSITIVE

BREAST CANCER

\section{Laura Dalimonte}

Odette Cancer Centre, Toronto, Canada

\section{Purpose}

To develop a decision aid (DA) prototype for post lumpectomy women 70 years and older with Stage I hormone sensitive invasive breast cancer, and to conduct a preliminary testing of the DA to ensure that the format and 
information presented is clear and acceptable to patients.

\section{Methods and materials}

During the developmental phase of a prototype of the DA, we incorporated feedback from the focus groups that were conducted with 16 older women with Stage I breast cancer. In addition, we applied the Ottawa Decisional Support Framework to ensure that the information on the DA was evidence-based, practical, and understandable by the patients. The final format of the DA was evaluated in one-on-one interviews with 12 Stage I, breast cancer patients in the same age group. All the participants completed their adjuvant radiation therapy at the Odette Cancer Centre. After the interviews, the participants received a pocket size version of the DA and an acceptability questionnaire. The acceptability questionnaire investigated patients' understanding of the information provided, length of the DA, its visual appearance and the graphics. In addition, patients were encouraged to provide any further comments regarding the format and content of the DA prototype.

\section{Results}

All participants rated the DA as extremely acceptable, found the format and content clear and informative and had desired such a tool when they were facing their treatment decisions. Four (4/12) women felt that although the DA booklet thoroughly presented all pertinent information, the content should be better organized. In addition, some women (4/12) felt that the language of the DA needed to be clarified and simplified for the lay audience who would be using the tool. The biggest concern from all participants was the timing of the delivery of the tool. Many women expressed the need for the tool to be administered prior to surgery or immediately after to allow for time to process the information presented in the DA and to generate questions for physician consults.

\section{Conclusions}

Based on patients' feedback, we revised the content of the DA. We also simplified the lan- guage in several parts of the DA that was suggested by the participants. The detailed analysis of the acceptability questionnaires will be presented at the meeting. The findings of this study indicate that older breast cancer patients' informational needs are complex. The DA can be a helpful educational tool in the decisionmaking process for treatment in this group of low risk breast cancer patients.

\section{WHAT ARE MY OPTIONS FOR BREAST CANCER TREATMENT? AN INFORMATIONAL NEEDS ASSESSMENT STUDY FOR OLDER WOMEN WITH STAGE I BREAST CANCER}

\section{Laura Dalimonte}

Odette Cancer Centre, Toronto, Canada

\section{Purpose}

To conduct a needs assessment from the patients' perspective for designing a decision aid (DA) and to encourage the development of DA's to help women prepare for the implications of their choices.

\section{Materials and Methods}

The study participants included 16 women over the age of 70 years treated at the Odette Cancer Centre. Women were invited to participate in one of six focus group discussions following their radiation therapy treatment for Stage I breast cancer. The main areas covered were related to the participants needs about; being diagnosed with breast cancer, treatment options available, making decisions about treatment, unexpected changes/experiences, advice for health-care professionals and preferences for receiving information. Discussions were audio recorded and professionally transcribed. The text was segmented and grouped according to each discussion topic.

\section{Results}

Many women identified several challenges in the decision-making process and identified 
different needs for making decisions about their treatments. Fifty percent of participants were not informed about possible treatment options by their physicians and were not fully engaged in choosing their treatments. In addition, they highlighted the informational needs that were helpful to make informed treatment choices. These needs were related to asking and discussing more specific questions during initial consultation, the possibility of using decisional aids such as diagrams or lists of online resources, a list of the side effects of radiation therapy and hormone treatment, what to expect when treatment is completed, and information about clinical trials and supportive services such as parking, volunteer drivers, educational materials for family members about treatment, and posttreatment follow-up.

\section{Conclusion}

The findings of this study indicate that older breast cancer patients' informational needs are complex. Ways of enhancing patients' involvement in the treatment selection process requires individualisation.

\section{RADIOGRAPHER INTER-OBSERVER VARIABILITY IN THE ASSESSMENT OF TOMOTHERAPY MEGA-VOLTAGE CT (MVCT) VERIFICATION IMAGES: A COMPARISON OF DIFFERENT ANATOMICAL SITES}

\section{Christian Day, Mark Elsworthy}

BUPA Cromwell Hospital, London, UK

\section{Introduction}

The inter-observer variability of image registration (IR) capabilities among a group of therapy treatment radiographers $(n=7)$ using the TomoTherapy Hi-Art ${ }^{\mathrm{TM}}$ system was measured for different anatomical sites.

\section{Methods and Materials}

Each radiographer retrospectively reviewed MVCT images off-line using the treatment operator workstation of the TomoTherapy
Hi-Art ${ }^{\mathrm{TM}}$ system. Five separate patients' images were reviewed for each anatomical group, these being Head \& Neck, Breast, Brain and Lung. One image per patient was reviewed by each radiographer, resulting in a total of 140 image registration assessments. Patients were selected at random from previously treated patients and the same images were assessed by each radiographer. Radiographers were not timed during IR and were not restricted in their IR technique. Variability was measured against the IR outcome of the lead radiographer whose assessments are considered as the gold standard.

Results

The mean inter-observer variability was found to be approximately $1 \mathrm{~mm}$ for all sites in the anterior/posterior and medio/lateral directions. Approximately $2 \mathrm{~mm}$ variability was detected in the superior/inferior direction for all sites except Head \& Neck which was observed to be $1 \mathrm{~mm}$.

\section{Conclusions}

The low inter-observer variability observed amongst this group of radiographers is reassuring and can help to inform future work practice with the confidence that a consistent standard is being met for IR across different anatomical sites.

AN AUDIT OF SET UP ERRORS FOR
BLADDER PATIENTS TREATED
WITH THE TOMOTHERAPY
HI-ARTTM SYSTEM TO
DETERMINE IMAGING FREQUENCY

Mark Elsworthy ${ }^{1}$, Andrew Garton ${ }^{2}$, Emma Keggin ${ }^{2}$

${ }^{1}$ Radiotherapy Department, ${ }^{2}$ Medical Physics Department, Bupa Cromwell Hospital, London

\section{Introduction}

An audit of set up correction data for a group of patients receiving tomotherapy for carcinoma of the bladder has been performed to determine 
a scanning frequency for treatment verification purposes.

\section{Methods and materials}

Setup correction data of 13 patients (365 treatment fractions) were retrospectively analysed. Patients were imaged daily and treated using the TomoTherapy Hi-Art system ${ }^{\mathrm{TM}}$ and were requested to have an empty bladder, no rectal preparation advice was provided. A systematic setup error (SSE) correction based on the average correction applied over the first 10 fractions was applied to subsequent fractions to determine both the systematic and random error components of the residual set up correction.

\section{Results}

Seven patients $(53.8 \%)$ would have completed treatment without a further setup correction. The remaining six patients $(46.2 \%)$ would have been considered for a further correction. Of the 235 post correction fractions analysed, $189(76.2 \%)$ aligned within $5 \mathrm{~mm}$. Alignments $>10 \mathrm{~mm}$ were associated with three patients and were seen in $10(4.3 \%)$ fractions. The mean setup errors was found to be $-0.67 \mathrm{~mm}$ $(\mathrm{SD} \pm 0.56 \mathrm{~mm})$ in the lateral direction, $-0.92 \mathrm{~mm}(\mathrm{SD} \pm 1.37 \mathrm{~mm})$ in the longitudinal direction and $-0.39 \mathrm{~mm}(\mathrm{SD} \pm 1.54 \mathrm{~mm})$ in the vertical direction. Random setup errors were $\pm 2.26 \mathrm{~mm}, \pm 1.83 \mathrm{~mm}$ and $\pm 2.8 \mathrm{~mm}$, respectively.

\section{Conclusions}

This audit of bladder setup corrections using a scenario of correcting the SSE after 10 fractions has shown that patients can safely proceed to weekly imaging. Daily imaging over the first 10 fractions allows time to assess setup reproducibility and bladder status.

\section{HEALTHWISE IT IS EASIER THAN YOU THINK?}

\section{Katie Hopkins} Cancer Information manager, Barnsley NHS
Foundation Trust, UK
The Cancer Services Department launched a Mobile Cancer Information Service in January 2010 , to enable us to educate the local population of Barnsley about cancer prevention and Awareness.

NHS Barnsley agreed to fund the service. Together building on the delivery and service development in partnership with NHS Barnsley, Barnsley MBC, Fit for the Future, Healthy Schools and the North Trent Cancer Network.

The main focus of the Barnsley Cancer Information Service is on education - 'To prevent the event'.

A Mobile Cancer Information Service - has the ability to focus on prevention within the community and deliver education in a variety of locations.

A structured programme for the Service will ensure young people; the general public, existing patients and their families/carers are adequately educated in relation to Cancer prevention and progression.

\section{Focus on the general public}

The Cancer Information Manager will take the mobile unit into popular public areas with to offer advice and information to the public.

Focus on young people

The service will visit schools, to deliver a structured education programme in line with the PSHE national curriculum.

Anti-smoking - Lung/Upper GI/Cervical/ Urological/Sex education - Cervical/Urology (HPV vaccination programme)

Alcohol - Upper and lower gastrointestinal

Sun exposure - Skin

Results

In the last 7 months the service has visited a number of different locations within Barnsley including Town centre, local supermarkets, Gyms and Schools delivering a programme in 
line with local and national cancer awareness initiatives. To date we have provided education and support to over 2000 people within Barnsley.

\section{BLADDER 'PLAN OF THE DAY' (IMPLEMENTATION OF ADAPTIVE RADIOTHERAPY FOR BLADDER CANCER)}

\section{Daniel Hutton, Angela Heaton, Pooja Jain}

Clatterbridge Oncology Centre, Liverpool, UK

\section{Objective}

Evaluate the feasibility of image-guided adaptive radiotherapy for bladder cancer patients at Clatterbridge Centre for Oncology. Conduct radiographer training to ensure competence in soft tissue bladder matching.

\section{Methodology}

Ten bladder patients were treated with radiotherapy using the cone beam computed tomography (CBCT) verification. CBCT scans were performed for the first three fractions than weekly.

The adaptive $\mathrm{RT}$ radiographers undertook an in-house training course comprising of anatomical lectures, practical sessions with CBCT registration software and a competency assessment.

Image registration was performed online by therapy radiographer using three different registration methods: manual bone match, manual soft tissue match and an automatic PTV match. Isocentre corrections were performed using the manual bone match with a $5 \mathrm{~mm}$ action level.

In-room timings were performed for this cohort and a control group of non-CBCT patients to allow comparison and assess departmental resource requirements.

\section{Results}

The CBCT registration results using the three methods agreed to within $5 \mathrm{~mm}$. Changes in bladder volume were observed over the course of treatment for the majority of patients. The planned PTV volume at times provides generous coverage that potentially could be reduced if the patients were replanned.

The in-room linac time for the online CBCT registration technique increased by $3 \mathrm{~min}$, compared to the non-CBCT technique.

\section{Conclusion}

Patients may benefit from a library of plans were a 'plan of the day' adaptive technique is employed to ensure PTV coverage. An additional benefit would be the reduction in normal tissue volume and the theoretical reduction in treatment related toxicities.

Therapy radiographers, with appropriate training have the skills and confidence to soft tissue match and evaluate PTV coverage and select the appropriate plan.

\section{CYBERKNIFE: THE CHALLENGES OF A NEW TREATMENT MODALITY}

\section{Stuart McCaighy}

\section{The London Clinic, UK}

This presentation seeks to outline and discuss in detail the local experience at the London Clinic in the successful implementation and running of a CyberKnife Radiosurgical Robotic System within a brand new radiotherapy department. The unique challenges of CyberKnife will be identified with reference to UK legislation and international radiosurgical practice. The presentation will cover all aspects of the patient's journey from selection to treatment delivery. Focus will be given to the needs of radiographer training, close working with medical physics and the importance of multidisciplinary working to provide a seamless service. CyberKnife's role within the department will be discussed along with our plan for the future in terms of new patient groups to be potentially treated, research and 
raising the profile of CyberKnife within the radiotherapy community and beyond.

This presentation will demonstrate how The London Clinic was able to successfully implement the CyberKnife unit within a very tight timescale and will offer a comprehensive insight to those centres that are considering investing in this new and exciting treatment modality and will also offer ideas and suggestions to departments who may be facing similar challenges in the future.

\section{D PLANAR IMAGING VERSUS 3D CONE BEAM CT FOR IMAGE GUIDED HEAD AND NECK RADIOTHERAPY \\ Ros Perry, A Poynter, H James, D Emmens, S Isherwood, R Parker, CD Scrase \\ Ipswich Hospitals NHS Trust, UK}

At Ipswich Hospital NHS Trust we have routinely treated all radical radiotherapy head and neck cancer cases using image-guided techniques since early 2009. Current practice is to use two orthogonal $\mathrm{kV}$ planar images to match the bony anatomy position, and correct for any isocentre discrepancy position before the treatment is delivered.

There are potential limitations, however, to the amount of information 2D planar imaging can give, making it difficult to detect out of plane rotations. In addition, although it is possible to detect shifts in the bony anatomy, it is not always easy to appreciate why these changes have occurred, nor the dosimetric or clinical consequences of them.

Our treatment equipment can also be used to acquire a 3D cone beam computed tomography (CBCT), giving the potential for image guidance using a full 3D data set including soft tissue structures. This also has the potential to be used for adaptive radiotherapy.

We have just opened recruitment to a single centre randomised trial comparing the isocentre position obtained between each of these methods; i.e., 2D planar imaging and 3D CBCT.

We are aiming to recruit 15 participants, each of whom will receive daily IGRT, by means of conventional 2D imaging but in whom once a week a CBCT will be obtained. The results of any difference in the isocentre position will be statistically analysed. Additional information collected will help determine the potential benefits of adaptive radiotherapy in this patient group.

An update on study progress and any interim results will be given.

INVESTIGATING JOURNAL READING

AND LITERATURE SEARCHING

PRACTICES OF RADIATION

THERAPISTS: AN EVALUATION OF

THE CHALLENGES AND BARRIERS

TO IDENTIFYING EVIDENCE FOR

PRACTICE DEVELOPMENT

Heidi Probst ${ }^{1}$, Angela Eddy ${ }^{1}$,

David Eddy ${ }^{1}$, Daniel Edwards ${ }^{2}$

${ }^{1}$ Sheffield Hallam University, UK, ${ }^{2}$ Cambridge

University Press, UK

\section{Introduction}

There is anecdotal evidence from journal reviewers that publishing authors have limited understanding of how to select appropriate keywords when submitting their work for publication or how the structure and content of the Abstract influences reader choice for downloading their work. The choice of keywords specifically is critical in ensuring that the work is easily retrievable using standard search terms utilised across a range of databases. In addition, it is not clear what the level of literacy searching skills in radiographers (or radiation therapists) is with limited research in the published literature to identify general skill levels in this area. This study aimed to identify the key strategies radiation therapists use when searching for evidence from the literature and how extensive journal article reading is across postgraduate therapists. 
In particular, the focus of the study was how practitioners choose search terms as part of a general search strategy. The results of this study will inform authors publishing in key radiotherapy journals in order to maximise the potential of their work being retrieved by interested parties.

\section{Method}

In order to gain some understanding of where practitioners start when searching for evidence an online survey was designed for a sample of radiation therapists to establish quantitative data on searching practices. The questions were based on key themes from the literature. In addition, a specific research question was posed and participants were asked to state what keywords they would use to search for evidence and to identify the key databases they would employ to retrieve relevant evidence as well as filtering techniques employed to select relevant articles.

\section{Significance of the research}

The study is expected to contribute knowledge and understanding to help authors disseminate their work more effectively to the practicing community, thus increasing user-pull methods of dissemination. It may also identify skill deficiencies in searching for evidence that will inform future training packages.

\section{THE ROLE OF AN INFORMATION} AND SUPPORT RADIOGRAPHER IN A BRAND NEW PURPOSE BUILT PRIVATE DEPARTMENT

\section{Helen Sales}

The London Clinic, UK

I will discuss the challenges that myself and the centre have encountered during the past 6 months in establishing a gold standard support and information service for a market savvy audience. Clients include renowned consultants, self paying, embassy and insurance funded patients, as well as freelance specialists.
To be ready and prepared to open our doors to the a fore mentioned group in under 4 months, we had to conduct audits and studies on what was needed, what was already being offered and what we could improve on. Looking at training issues for state of the art equipment, e.g., CyberKnife, RapidArc, cone beam CT, IMRT, and IGRT. We also had to develop a strong staff group and address the issues surrounding bringing together a wide range of people with a wealth experiences to enhance team work and facilitate optimum working levels.

I would also like to explore our developed patient pathway, links with members of the multidisciplinary team and communications with staff further afield. I will discuss issues such as working in a paperless department and how we have developed patient care.

\section{DOSIMETRIC IMPLICATIONS OF WEIGHT LOSS ON HEAD AND NECK CANCER PATIENTS \\ C Smith, J Stratford, M Duffy, S Chauhan, J Parker}

Wade Centre for Radiotherapy Research, The Christie Hospital NHS Trust, Manchester, UK

\section{Introduction}

With increasing use of chemoradiotherapy regimes, weight loss is a common problem in head and neck radiotherapy resulting in potential dosimetric implications to the treatment plan. A local audit was undertaken to assess the impact of weight loss on the treatment plan for head and neck cancer patients.

\section{Methods}

Between November 2006 and December 2009 data was collected for 20 patients. On-treatment setup displacements were measured from x-ray volumetric images (XVIs) acquired using cone beam computed tomography (CBCT) from an Elekta Synergy linear accelerator. A no action level (NAL) protocol was used to correct systematic displacements over $0.3 \mathrm{~cm}$ based on 
the first three fractions. Shell fit and visual assessment of the patient were used in conjunction with CBCT to assess the impact of weight loss on the treatment plan.

\section{Results}

27 XVIs (20 patients) were sent for planning review to assess the impact of weight loss. $1 / 20$ patients required a total re-plan. $1 / 20$ patients required a monitor unit recalculation. 18/20 patients had less than $2 \%$ change in dose throughout the treatment volume and to critical organs at risk; therefore no correction was necessary to the treatment plan.

\section{Conclusion}

CBCT has provided us with the means to assess and adapt the treatment plan for the impact of weight loss. This audit demonstrates the robustness of the IMRT class solution currently used within this institution as only $2 / 20$ patients required planning intervention. Future advances in IMRT treatment planning incorporating further margin reduction and dose escalation will require $\mathrm{CBCT}$ to ensure safe and effective treatment delivery.

\section{References}

1. Mangar $\mathrm{S}$ et al. Evaluating predictive factors for determining enteral nutrition in patients receiving radical radiotherapy for head and neck cancer: A retrospective review. Radiother Oncol 78 (2006) 152-158.

HPC CPD AUDIT - ONE RADIOGRAPHERS EXPERIENCE

\section{Alexandra Taylor}

\section{St Fames's Institute of Oncology, Leeds, UK}

Having been audited by the HPC this year this presentation is aimed at helping fellow radiographers who may be selected in the future.

This will start with how I felt on initially being selected and also how I then dealt with the fact that my first submission was rejected.
The body of the presentation will offer advice on where to start with putting your submission together, such as finding out what help is available and what support your department can offer. It is important to look through your CPD folder and assess what have you got so far and are there any gaps that need filling in such as half written reflections etc.

Sit down with the standards provided by the HPC and work out what evidence you have to achieve each of the standards.

This will be followed by a discussion about what exactly the HPC require. This includes a personal statement, a 1,500 word piece acting as your supporting statement. In this you should describe what evidence you are submitting to meet the standards required. The evidence you are submitting should then be summarised in a table at the end of your statement and the actual content attached as an appendix.

The presentation will finish with an overview of the main things to remember and also some extra hints and tips. When you get selected don't panic, odds are you have everything you need it will just be a matter of 'sprucing it up a bit'.

\section{MICROBUBBLE-ULTRASOUND \\ RADIOENHANCEMENT OF BLADDER CANCER TREATMENT William Tran \\ Sunnybrook Health Sciences Centre, Toronto, Canada}

\section{Background}

Traditionally used in ultrasound imaging as contrast agents, microbubbles are small microspheres, composed of either a lipid or protein shell that encapsulate various gasses. When exposed to certain ultrasound frequencies, microbubble agents oscillate and can burst. In effect, this biophysical phenomenon has been demonstrated to show endothelial cell perturbations in tumour vasculature and therefore causing tumour shrinkage. Our preliminary studies 
indicate that these tumours are also exhibiting enhanced radiosensitivity.

\section{Objectives}

To demonstrate the potential effects of microbubbles as radio-sensitizing agents in tumour vasculature and as anti-vascular/anti-angiogenic agents in cancer therapeutics.

\section{Methods}

Human bladder carcinoma xenografts were inoculated into SCID mice and observed for tumour growth. Tumours were allowed to grow approximately $7 \mathrm{~mm}-10 \mathrm{~mm}$ in diameter and were treated with nine conditions of combined ultrasound and varying levels of no, low and high concentrations of microbubbles. Tumours were imaged with ultrasound before and after treatment and sacrificed 24 hours post-treatment. Tumours were analyzed using immunohistochemical techniques in conjunction with ultrasound imaging technology.

\section{Results}

Our results show preliminary data that bladder cancer xenografts are responsive to microbubble treatments when combined with ultrasound targeting and radiation. Immunohistochemical analysis demonstrates apoptotic regions within tumour and demonstrate specific foci in tumour vascular ablation.

\section{Conclusions}

Microbubbles, when combined with ultrasound and radiation may be potential anti-vascular agents in anti-tumour therapeutics.

\section{ADVANCED PRACTITIONER ROLE- WHERE? WHY? HOW? SO WHAT?}

\section{Jo Treeby}

Addenbrooke's Hospital, Cambridge, UK

With the introduction of the four tier structure and acknowledgement of the need for expert practice, radiographers now perform tasks that traditionally have been the responsibility of physicians. In his 2007 paper, 'Getting it right for people with cancer: Clinical case for change' Professor Mike Richards, National Cancer Director for England recognised that adapting staff roles gives more flexibility and an improvement in service.

The role of Advanced Practitioner Urology Radiographer was created to meet local service need, an important task being to conduct ontreatment review clinics unsupervised. Skills acquired through training schemes promoted medical staff confidence in radiographer competence allowing role expansion to encompass post-treatment clinics (including psycho-sexual counselling and erectile dysfunction), taking treatment consent, research and audit. In prostate brachytherapy there is participation in the implant process, managing post-implant toxicity and being contact point for brachytherapyrelated matters for physicians and others.

A major aim of advanced practice is enhancement of patients' treatment experience. Audit evaluating patient satisfaction with radiographer led review clinics showed a very high level of approval, demonstrating that changing responsibilities within the radiotherapy pathway can enrich patients' treatment experience. The Advanced Practitioner Role can motivate and promote job satisfaction, but may have implications for those treating patients on a daily basis who should not be regarded as being less accomplished. It is essential that radiographer treatment teams continue to be recognised as the skilled and professional practitioners who encompass the heart of the radiotherapy service.

FIDUCIAL MARKERS AND PERIPATETIC PROSTATES - A SIMPLE SOLUTION FOR A COMPLEX PROBLEM?

\section{Jo Treeby}

Addenbrooke's Hospital, Cambridge, UK

\section{Introduction}

Prostate movement is a recognised and welldocumented phenomenon. Traditionally accuracy 
of prostate radiotherapy has been assessed retrospectively using images based on bone anatomy without evidence of current prostate position. Prostate movement between treatment fractions imposes limitations of effectiveness which are self evident. As departments move to IMRT and consider reducing treatment margins it is essential that movement is taken into account to minimise the risk of missing what is in effect a moving target.

Daily imaging of radio-opaque markers within the prostate permits isocentre repositioning to correct for prostate positional variations prior to each treatment.

\section{Method}

Following a successful pilot project involving 6 patients, a study of 50 patients was undertaken. Gold, non-migratory seeds were implanted in the prostate of patients shortly before radical radiotherapy. Seeds were identified on daily portal images and online analysis of seed position was performed using Isoloc software to measure out-of-plane rotations previously not accounted for in two-dimensional portal imaging systems. Automated three-dimensional vector and rotational displacements were generated: from these the necessary couch movements to correct the isocentre position were determined and made before treatment was delivered. No extra radiation was delivered to achieve images.

PROMOTING A RESEARCH CULTURE: IDENTIFYING THE CHALLENGES AND OPPORTUNITIES FACED BY RADIATION THERAPISTS IN INITIATING AND DEVELOPING RESEARCH

\section{PROJECTS}

\section{Angela Turner}

\section{Odette Cancer Centre, Toronto, Canada}

The presentation will describe our experience and lessons learned during a research project we undertook to identify opportunities and challenges for Radiation Therapist's participation in research at our centre.

\section{Objectives}

At the end of this session, participants will be able to:

1. Discuss the application and approach to focus group data collection (including pre -group preparation, facilitation of groups, data analysis, etc.).

2. Develop strategies and initiatives to encourage implementation of research programs among health-care workers through illustrations presented from the results from our project.

\section{Outline}

I. Background and rationale to our project

II. Conducting focus groups; how when and why ... and why not!

III. Data analysis in qualitative research: what do the results mean?

IV. Results of our project. Lessons learned from our data

V. Strategies to develop research initiatives at our centre (which can be translated to other health-care facilities).

AN EVALUATION OF THE SUPRACLAVICULAR AND AXILLARY LYMPH NODE RADIOTHERAPY PLANNING TECHNIQUE

\section{Camarie Welgemoed}

Imperial College Healthcare NHS Trust, London, $U K$

The study aims to evaluate the current departmental supraclavicular (SC) and axilliary (AX) lymphnode (LN) radiotherapy planning technique by answering two questions: 
Does the anterior/posterior radiotherapy boost technique and conventional field border definition provide sufficient dose coverage of the SCF and AX LN's?

What radiotherapy dose is delivered to the brachial plexus (BP) volume, using an anterior/posterior boost technique?

Evidence suggests that the position of SC and AX LN's changes with a change in arm position. The effect of this change has not been investigated before replacing conventional simulation with $\mathrm{CT}$ simulation. Consequently field borders are determined by using twodimensional landmarks and the dose calculated as for conventional planning.

In view of existing evidence, the author decided to evaluate present departmental practice in a retrospective study, utilising the information provided by current technology. The study includes 54 patients who previously underwent CT simulation, positioned on an Exact Med-Tech breast board with the ipsilateral arm raised and supported by an arm rest. After completion of the CT scan, geometry of the irradiation fields was defined on Prosoma virtual simulation software. The LN groups and BP were contoured retrospectively. By reproducing the original treatment planning technique and parameters on the treatment planning system, dose received by the SCF LN's, AX LN's and BPs has been evaluated using dose-volume histogram (DVH) information.

It is hoped that results from this study will determine the accuracy of the current technique, facilitate standardisation in field border definition inform future technique decision making.

\section{WHAT WERE OUR EXPECTATIONS OF RADIOGRAPHER LED BREAST MARK- UP SEVEN YEARS AGO, COMPARED TO TODAY?}

\section{Camarie Welgemoed}

Imperial College Healthcare NHS Trust, London, $U K$
What were our expectations of radiographer led breast mark-up seven years ago, compared to today?

This is a reflection on progress in radiographer led breast planning over the last 7 years. The discussion includes initial expectations and main achievements from both a service manager and radiographer's perspective and raises the question as to whether patients receiving radiotherapy treatment for breast cancer received any benefits from the resulting service changes.

In an aim to reduce simulation session delays, seven years ago, three radiographers attended a breast planning and prescribing course. As a result management hoped to reduce the waiting list, improve staff retention and increase radiographer knowledge. As one of the radiographers concerned I was keen to improve my knowledge, pass the course and complete competencies in clinical breast mark up. At this time I began to recognise the opportunity to further develop my role in future, perhaps a consultant radiographer role.

Management expectations were exceeded. The patient pathway improved, there were substantial technique improvements, audit was imbedded in departmental culture and benefit gained from staff training. Although from a radiographer's perspective the emphasis on achievements is different, patients receiving radiotherapy for breast cancer benefit directly from these practice developments which include immobilisation improvements, technique development, treatment verification changes and radiographer led research.

Perhaps this example of developing the radiographer led breast planning role to consultant level will encourage others to take on the challenge with more managers raising their future expectations of the radiographer led breast mark-up role. 